\title{
Notas sobre la dieta, desarrollo de jóvenes y distribución del Búho Estigio Asio stygius (Strigiformes: Strigidae) en Ecuador
}

\author{
Héctor Cadena-Ortíz ${ }^{1,2, *}$, Jorge Bedoya ${ }^{1,3}$, Glenda M. Pozo-Zamora ${ }^{1,2}$, Julie Watson ${ }^{1}$, Jorge Brito ${ }^{2,4}$ \\ ${ }^{1}$ AvesQuito (Club de Observadores de Aves), Ecuador \\ ${ }^{2}$ Museo Ecuatoriano de Ciencias Naturales del Instituto Nacional de Biodiversidad, División de Ornitología y Mastozoología, Calle Rumipamba 341 y \\ Av. de los Shyris, Casilla 17-07-8976, Quito, Ecuador \\ ${ }^{3}$ Yaku Parque Museo del Agua, Calle el Placer Oe127, Quito, Ecuador \\ ${ }^{4}$ Instituto de Ciencias Biológicas, Escuela Politécnica Nacional, PO Box 17-01-2759, Quito, Ecuador. \\ *Autor para correspondencia, e-mail: fercho cada@yahoo.es \\ Editado por/Edited by: Diego F. Cisneros-Heredia \\ Recibido/Received: 12 Diciembre 2016. Aceptado/Accepted: 21 Junio 2018. \\ Publicado en línea/Published online: 13 Septiembre 2018.
}

\section{Notes on the diet, juvenile development and distribution of the Stygian Owl Asio stygius (Strigiformes: Strigidae) in Ecuador}

\begin{abstract}
Resumen
Presentamos el primer aporte al conocimiento sobre la dieta del Búho Estigio Asio stygius en Ecuador. El estudio se realizó a partir de 38 egagrópilas y $127 \mathrm{~g}$ de material disgregado colectado en dos localidades urbanas y una rural de la provincia de Pichincha entre 2015 y 2016. Encontramos 157 ítems-presas agrupadas en 8 taxones. Las aves fueron predominantes en la dieta de este búho, numéricamente y en biomasa, por lo que consideramos a A. stygius como un depredador especialista en aves. Adicionalmente, en esta publicación presentamos información sobre el desarrollo de dos jóvenes de este búho, y compilamos los registros de distribución existentes de $A$. stygius en Ecuador, los cuales están principalmente concentrados en la provincia de Pichincha.
\end{abstract}

Palabras clave: Egagrópilas, Ecuador, plumaje, ocurrencia.

\begin{abstract}
We present the first contribution to the knowledge about the diet of the Stygian Owl Asio stygius in Ecuador. We studied 38 pellets and $127 \mathrm{~g}$ of disaggregated material collected in two urban and one rural localities in the province of Pichincha between 2015 and 2016. We found 157 prey-items grouped in 8 taxa. Birds were prevalent, numerically and in terms of biomass, thus we consider A. stygius as a bird specialist predator. Additionally, herein, we present information on the development of two juvenile Stygian owls, and compile distribution records of A. stygius in Ecuador, which are main concentrated in the province of Pichincha.
\end{abstract}

Keywords: Ecuador, pellets, plumage, occurrence.

\section{INTRODUCCIÓN}

La descripción de la dieta de una especie es un componente importante para el entendimiento de su ecología. El conocimiento de la ecología trófica de aves rapaces nocturnas del Ecuador es escaso (Freile et al., 2012; CadenaOrtiz et al., 2013), a pesar de recientes publicaciones que analizan la dieta de algunas especies (Brito et al., 2015; Cadena-Ortiz et al., 2016; Pozo-Zamora et al. 2017). Esta falta de información puede deberse a la baja densidad poblacional y a los hábitos discretos y nocturnos de la mayoría de las especies de estrigiformes (Freile et al., 2012). Sin embargo, también hay limitantes metodológicas, como la falta de referencias para identificar contenidos estomacales y de egagrópilas y falta de esfuerzos de muestreo y publicación de datos (Cadena-Ortiz et al., 2013). 
El Búho Estigio Asio stygius se distribuye discontinuamente desde el norte de México al noreste de Argentina (König \& Weick, 2008). En Ecuador es una especie rara, registrada entre los 1700 a $3100 \mathrm{~m}$ en los Andes, en hábitats como bordes de bosque, áreas agrícolas, parques y plantaciones forestales (Ridgely \& Greenfield, 2001). Sobre la dieta de $A$. stygius se conoce que se alimenta de aves, murciélagos, ranas e insectos; siendo notable la ausencia de roedores dentro de su dieta (Lehmann, 1957; Borrero, 1967; Franz, 1991; Motta \& Taddei, 1992; Kirkconnell et al., 1999; Lopes et al., 2004; Motta-Junior, 2006; Phillips, 2011).

Hasta donde conocemos, esta es la primera contribución al conocimiento sobre la dieta de Asio stygius. En esta publicación adicionalmente presentamos las observaciones realizadas a dos jóvenes de este búho y compilamos los registros existentes para esta especie en el Ecuador.

\section{METODOLOGÍA}

Entre enero 2015 y agosto 2016 y de manera esporádica colectamos egagrópilas y material disgregado de Asio stygius en tres localidades del Distrito Metropolitano de Quito, provincia de Pichincha (Tabla 1): Parque Arqueológico Rumipamba, Parque Bicentenario y una propiedad privada en la parroquia rural de Nayón. Las dos primeras localidades son parques emplazados en la zona urbana y rodeados de edificaciones, mientras que el tercero se encuentra en una parroquia rural. El Parque Arqueológico Rumipamba tiene un área de 0,28 km2 y su vegetación está compuesta predominantemente por árboles de Eucaliptus sp. y pequeños remanentes de vegetación nativa. El Parque Bicentenario tiene un área de $1,38 \mathrm{~km} 2$, su escasa vegetación tiene mayor densidad en un arbolado de Ciprés Cupressus sp, y hasta febrero de 2013 fue el aeropuerto internacional de Quito. La propiedad privada en Nayón tiene un área de 1,9 km2, y donde se realizaron los muestreos predominan pastizales, árboles frutales dispersos de Inga insignis y Anonna chirimoya y remanentes de vegetación nativa, con quebradas pronunciadas que bordean al río Machángara.

Secamos las egagrópilas a temperatura ambiente sobre una placa metálica que absorbe calor solar durante dos días Para cada egagrópila tomamos medidas de longitud y ancho (en $\mathrm{mm}$ ) utilizando un calibrador digital (precisión $\pm 0,01 \mathrm{~mm}$ ), pesamos con una balanza analítica $(120 \mathrm{~g} \times 0,001 \mathrm{~g})$ y disgregamos manualmente. Para identificar las presas comparamos con especímenes del Museo Ecuatoriano de Ciencias Naturales del Instituto Nacional de Biodiversidad (MECN), donde también depositamos el material analizado en la sección de egagrópilas de la colección de Mastozoología. La masa promedio de las presas se obtuvo de las etiquetas de los especímenes de aves colectados en Quito y sus alrededores, y depositados en el MECN. Cuando no llegamos a una identificación específica, empleamos los promedios de las especies más comunes en el área detalladas por Cisneros-Heredia et al. (2015) de la siguiente forma: para los ítems agrupados en Parulidae se promedió los pesos de Reinita Coronirrojiza Myiothlypis coronata y Candelita de Anteojos Myioborus melanocephalus y para los paseriformes no identificados promediamos los pesos de los otros paseriformes-presa identificados. Para cada ítem-presa consumido calculamos el número mínimo de individuos (NMI), contando únicamente el número de pares de mandíbulas en insectos o restos de cráneos y húmeros en aves y mamíferos con el fín de evitar reconteo (Manning \& Jones, 1990). Obtuvimos la biomasa de cada ítem-presa multiplicando la masa promedio (en gramos) de cada especie por el NMI correspondiente (Herrera \& Jaksic, 1980).

Las observaciones realizadas a dos jóvenes de $A$. stygius se las efectuaron en la localidad de Nayón, el sitio fue visitado aproximadamente cada dos días para observar su comportamiento y fotografiarlos, las visitas fueron cortas - menos de una hora, para no perturbar al grupo familiar. Obtuvimos registros de A. stygius a través de redes sociales, incluimos los que tenían evidencia fotográfica o grabaciones de sus cantos, en ausencia de esto, consideramos los registros provenientes de observadores experimentados con las aves del país. Incluimos también registros obtenidos de las plataformas virtuales eBird (2016) y VertNet (2016), los de la primera coinciden con su distribución esperada y muchos presentan fotografías de respaldo. Los de VerNet, tienen especímenes de respaldo, sin embargo, son antiguos por lo que no se pudieron referenciar con certeza. También incluimos registros de nuestras visitas a las colecciones ornitológicas del Instituto Nacional Mejía - Museo de Ciencias Naturales (INM-MCN), Museo Ecuatoriano de Ciencias Naturales (MECN) y Museo de Zoología de la Pontificia Universidad Católica del Ecuador (QCAZ). 
TABLA 1. Detalle de localidades muestreadas para la dieta del Búho Estigio Asio stygius en Pichincha, Ecuador.

\begin{tabular}{|c|c|c|c|c|c|c|}
\hline Localidad & Coordenadas & $\begin{array}{l}\text { Elevación } \\
\text { (en m) }\end{array}$ & $\begin{array}{l}\text { Periodo de } \\
\text { permanencia }\end{array}$ & $\begin{array}{l}\text { Individuos } \\
\text { observados }\end{array}$ & Percha usual & $\begin{array}{l}\text { Egagrópilas } \\
\text { recolectadas }\end{array}$ \\
\hline $\begin{array}{l}\text { Parque } \\
\text { Arqueológico } \\
\text { Rumipamba }\end{array}$ & $0.180^{\circ} \mathrm{S}, 78.499^{\circ} \mathrm{O}$ & 2890 & $01 / 2015-01 / 2016$ & 1 & $\begin{array}{l}\text { A } 7 \text { m en un } \\
\text { Eucaliptus } \\
\text { sp. }\end{array}$ & $\begin{array}{l}3 \text { y } 77 \mathrm{~g} \text { de } \\
\text { material } \\
\text { disgregado }\end{array}$ \\
\hline $\begin{array}{l}\text { Parque } \\
\text { Bicentenario }\end{array}$ & $0.145^{\circ} \mathrm{S}, 78.485^{\circ} \mathrm{O}$ & 2800 & $02-04 / 2016$ & 1 & $\begin{array}{l}\text { A } 5 \text { m en un } \\
\text { Cupressus sp. }\end{array}$ & $\begin{array}{l}\text { Solo restos de } \\
\text { presas }\end{array}$ \\
\hline Nayón & $0.157^{\circ} \mathrm{S}, 78.415^{\circ} \mathrm{O}$ & 2350 & $09 / 04-20 / 05 / 2016$ & $\begin{array}{l}4 \\
\text { (2 adultos y } \\
2 \text { jóvenes) }\end{array}$ & $\begin{array}{l}1.5 \mathrm{~m} \text { en un } \\
\text { Inga } \mathrm{sp} .\end{array}$ & $\begin{array}{l}35 \text { egagrópilas } \\
\text { y } 50 \mathrm{~g} \text { de } \\
\text { material } \\
\text { disgregado }\end{array}$ \\
\hline
\end{tabular}

\section{RESULTADOS}

Determinamos el consumo de 157 ítems-presas agrupadas en 8 taxones (Tabla 2) a partir de siete restos de presas, $127 \mathrm{~g}$ de material disgregado y 38 egagrópilas (Tabla 1). Las egagrópilas presentaron las siguientes medidas: largo de 27-62 $\mathrm{mm}(41,3 \pm 7,8 \mathrm{~mm})$, ancho de 18-37 mm (25,3 \pm 4,2 mm), peso 1,2-6,8 g (3,3 $\pm 1,3$ g) y el número de presas por egagrópila fue $1-3$ presas (1,43 $\pm 0,6$ presas). Las presas encontradas se agrupan en aves, mamíferos e insectos. El grupo más consumido por Asio stygius fue el de las aves; los otros dos grupos representaron apenas el 13,4\% de frecuencia. La Tórtola Orejuda Zenaida auriculata fue el ítem preferencial en frecuencia y también el ítem que más aportó en biomasa (Tabla 2). En el Parque Bicentenario solo se colectaron restos de presas bajo la percha usual de A. stygius, que incluyeron cuatro Turdus fuscater, plumas de Zenaida auriculata y un Mimus gilvus. En esa misma percha se vio a un búho despojar el pico y extremidades a un individuo de T. fuscater a medio día (Fig. 1).

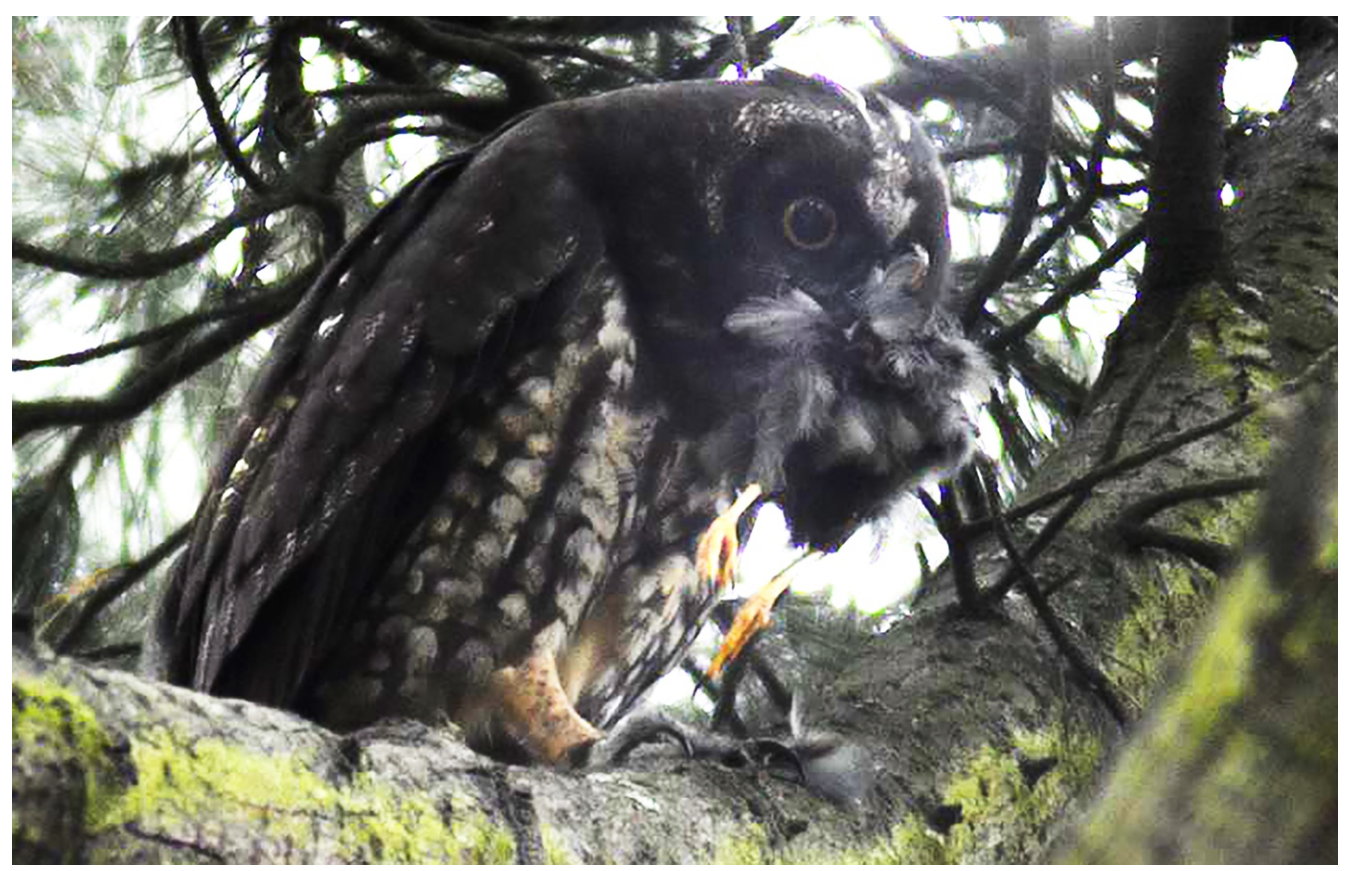

FIGURA 1. Búho Estigio Asio stygius depredando un Mirlo Grande Turdus fuscater a medio día en el Parque Bicentenario, 29 de febrero de 2016 (Fotografía: César Moreno). 
TABLA 2. Presas del Búho Estigio Asio stygius en Pichincha, Ecuador. Peso y biomasa (BM) en gramos; Número mínimo de individuos (NMI), Porcentaje (\%).

CLASE

Orden / Familia / Especie

Peso NMI $\% \quad$ BM $\quad \%$

\begin{tabular}{llllll}
\hline AVES & & & & & \\
Columbiformes / Columbidae / Zenaida auriculata & 113 & 116 & 73,9 & 13108 & 90,5 \\
Passeriformes / Turdidae / Turdus fuscater & 123 & 5 & 3,2 & 615 & 4,2 \\
Passeriformes / Mimidae / Mimus gilvus & 71 & 1 & 0,6 & 71 & 0,5 \\
Passeriformes / Parulidae & 11 & 3 & 1,9 & 33 & 0,2 \\
Passeriformes no identificados & 68 & 11 & 7,0 & 748 & 5,2 \\
MAMALIA & & & & & 0,2 \\
Chiroptera / Molossidae / Molossus rufus & 32 & 1 & 0,6 & 32 & 0 \\
INSECTA & & & & & 0 \\
Coleoptera / Dynastidae / Heterogomphus bourcierii & 0 & 18 & 11,5 & 0 & 0 \\
Coleoptera / Carabidae / Platicoelia lutens & 0 & 2 & 1,3 & 0 & 0 \\
TOTAL & & 157 & & 14480 & \\
\hline
\end{tabular}

En Nayón se pudo realizar observaciones regulares del cuidado parental de Asio stygius en un lapso de 35 días (16 abril al 20 mayo 2016) (Tabla 3). Durante ese período, no fue evidente un cambio marcado en la morfología de los juveniles. Sin embargo, las fotografías tomadas permiten ver un sutil cambio progresivo en su plumaje (Fig. 2). Los jóvenes presentaron el vientre barrado, el disco facial negro y las plumas que forman los penachos u "orejas" pequeñas. La presencia de plumón disperso en el cuerpo fue evidente por el aspecto esponjado de los jóvenes, y en la parte posterior de su cabeza presentaron plumas claras. Los últimos días que fueron observados, el plumaje del vientre se tornó más oscuro y en el pecho empezó a exhibirse líneas paralelas con diseño espina pez como en los adultos; el color de iris siempre fue amarillo.

TABLA 3. Observaciones a un grupo familiar de Asio stygius en Nayón durante el año 2016.

\begin{tabular}{lll}
\hline Fecha & $\begin{array}{l}\text { Hora de } \\
\text { visita }\end{array}$ & Actividad \\
\hline 9 y 11 Abril & $8 \mathrm{~h} 00$ & Adultos perchados \\
\hline 16 Abril & $8 \mathrm{~h} 00$ & $\begin{array}{l}\text { Dos jóvenes perchados en medio de dos adultos, ante nuestra presencia adultos vuelan } \\
\text { a perchas cercanas. }\end{array}$ \\
\hline 20 Abril & $18 \mathrm{~h} 00$ & $\begin{array}{l}\text { Adultos vocalizan e inician forrajeo minutos después ambos adultos entregan presas } \\
\text { (no identificadas) a los jóvenes. }\end{array}$ \\
\hline 7 Mayo & $12 \mathrm{~h} 00$ & Un adulto y un joven observados a 100 m de percha habitual. \\
\hline 20 Mayo & $8 \mathrm{~h} 00$ & $\begin{array}{l}\text { Jóvenes activos, cambiando rápidamente de rama en el mismo árbol, uno de ellos } \\
\text { extiende sus alas (interpretado como intimidación hacia observadores). Los padres se } \\
\text { encontraban en las cercanías. } \\
\text { Este fue el último día que fueron vistos los cuatro búhos en la localidad pese a } \\
\text { reiteradas visitas posteriores. }\end{array}$ \\
\hline
\end{tabular}




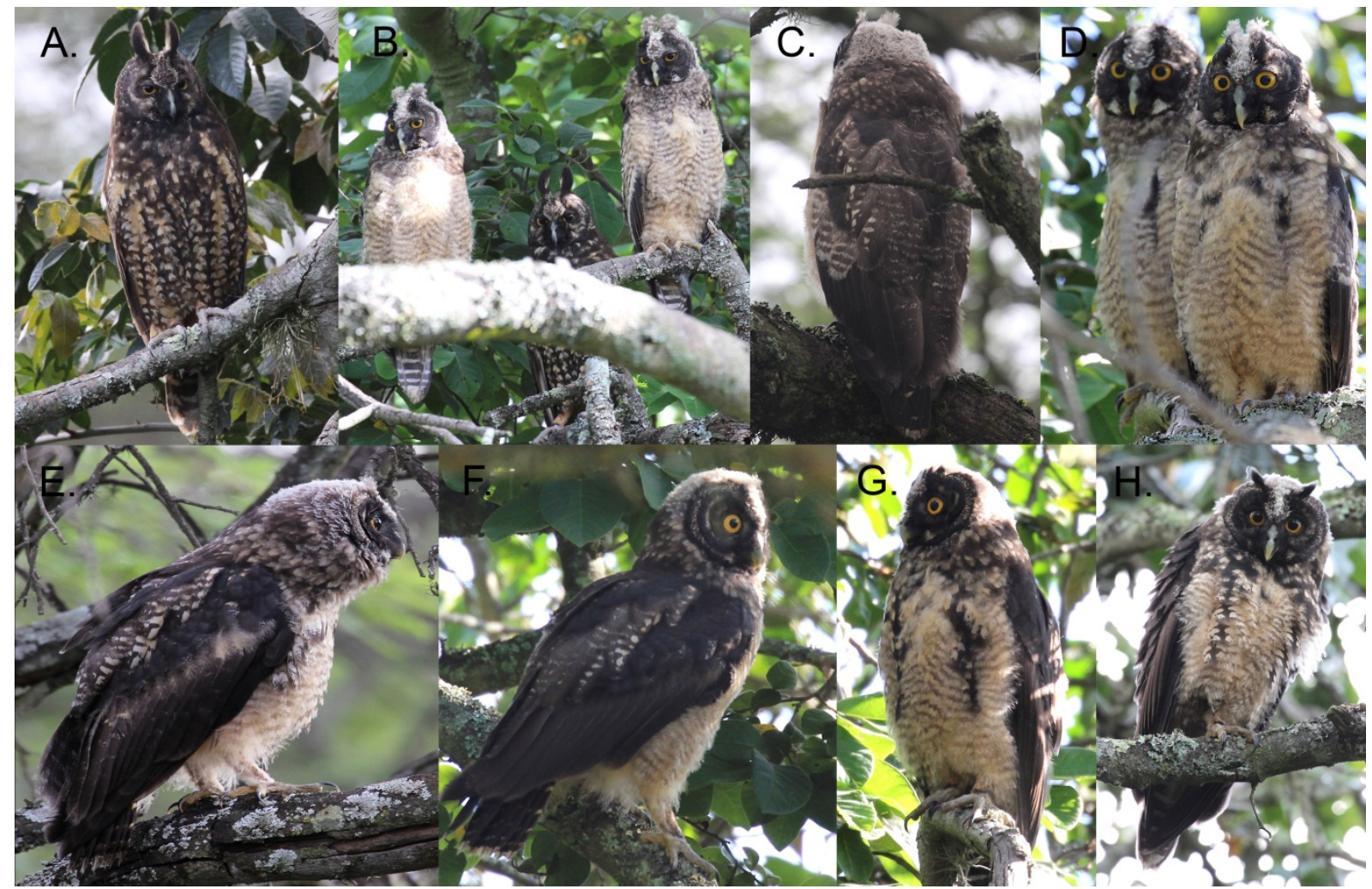

FIGURA 2. Grupo familiar del Búho Estigio Asio stygius observada en Nayón, Pichincha durante el 2016: A) Adulto, 24 abril; B) jóvenes y adulto en el fondo, 24 abril; C) dorso de joven, 30 abril; D) jóvenes, 3 mayo; E) joven, 7 mayo; F) y G) joven, 12 mayo; H) joven, 20 mayo. (Fotografías: Julie Watson).

En total reunimos registros de distribución de Asio stygius en 38 localidades en Ecuador, de las cuales 24 se emplazan en la provincia de Pichincha. Diecinueve registros son recientes, en los últimos 13 años, mientras que el resto supera los 24 años de antigüedad. Hay 21 especímenes de museo para $A$. stygius de Ecuador, de estos únicamente 5 reposan en colecciones nacionales (Tabla 4, Fig. 3).

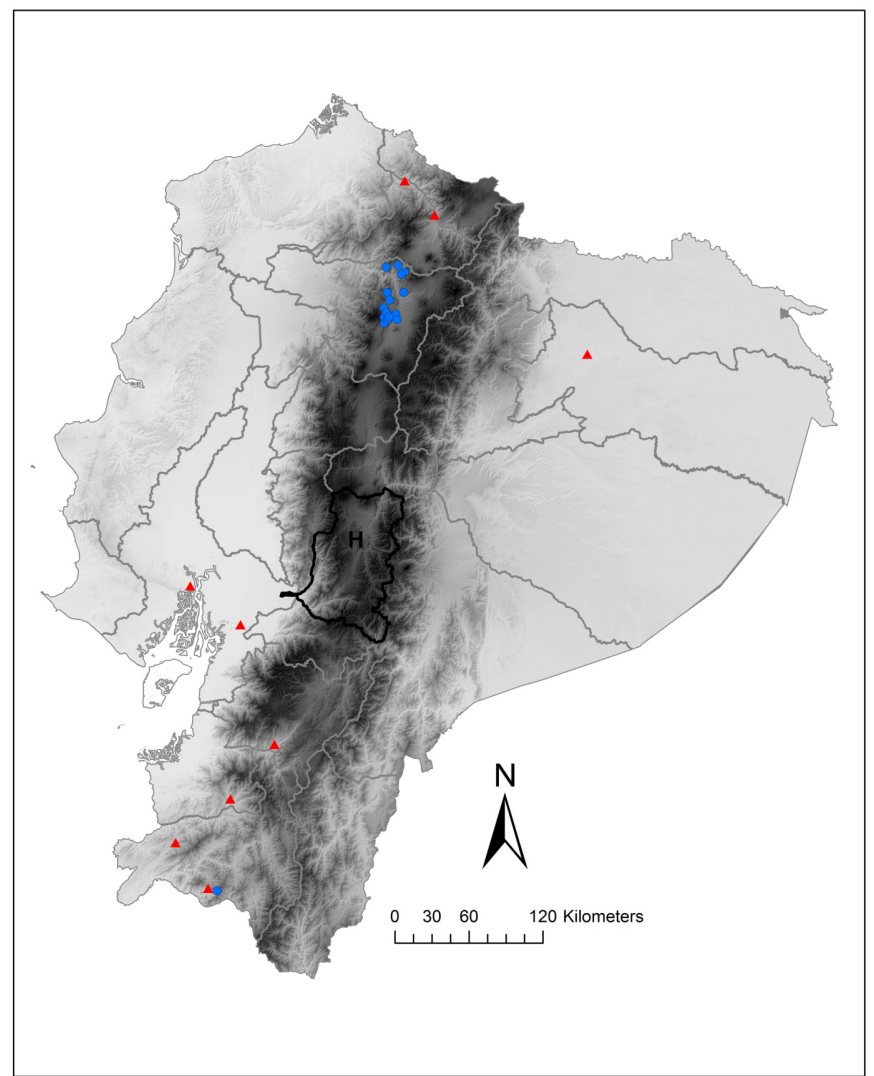

FIGURA 3. Distribución de Búho Estigio Asio stygius en Ecuador con los registros que se pudieron georeferenciar confiablemente. Círculos azules representan registros dentro del rango altitudinal esperado de la especie y triángulos rojos son registros bajo los $1500 \mathrm{~m}$. La provincia de Chimborazo presenta un registro histórico (ver Tabla 4). 


\section{DISCUSIÓN}

En base a nuestros resultados consideramos que Asio stygius - al menos en la provincia de Pichincha, actúa como un depredador especialista en aves y con táctica de caza oportunista. Especialista (de estrecho nicho trófico, Jaksic, 1989) porque apenas registramos el consumo de 9 ítems y oportunista (que ingiere las presas en las mismas abundancias relativas de su medio, Jaksic, 1989) porque la mayor frecuencia de presas encontradas en las egagrópilas 73,9\%, corresponde con una de las aves más abundantes en Quito: Zenaida auriculata (Cisneros-Heredia et al., 2015). Lo antagónico sería generalista, de amplio nicho trófico, y selectivo, que lleva a algunas o todas las presas en diferentes proporciones a las presentes en el área de caza (Jaksic, 1989). Franz (1991) menciona que $A$. stygius caza activamente al alba y crepúsculo, con capturas aéreas de murciélagos, aves e insectos grandes. Sin embargo, C. Moreno (in litt., 2016) observó y fotografió (Fig. 3) un individuo de $A$. stygius mientras despojaba el pico y extremidades a un individuo de Turdus fuscater a medio día, en el Parque Bicentenario. La predominancia en frecuencia de aves que encontramos en la dieta de Asio stygius de Pichincha (97.9\%), concuerda con estudios realizados en Planaltina, Brasil, donde se encontró únicamente aves entre los restos de presas de un nido (Lopes et al., 2004). En San Paulo, Brasil, la frecuencia de aves como presa de $A$. stygius representó entre el 90 y 92\% (Motta \& Taddei, 1992; Motta-Junior, 2006). En Cayo, Belice, las aves representaron el $62 \%$ de la dieta (Philips, 2011). Otros estudios señalan que los grupos predominantes de aves fueron semilleros 62.5\% (Motta \& Taddei, 1992), paseriformes 83.6\% (Motta-Junior, 2006) o colúmbidos (Lehmann, 1957; no presentó datos cuantitativos).

En nuestro estudio sólo una egagrópila presentó restos de murciélago, a pesar de que en otras localidades los murciélagos fueron un componente importante en la dieta de Asio stygius. Por ejemplo, se menciona predominancia de murciélagos en un número no especificado de egagrópilas tanto en los alrededores de Medellín, Colombia (Borrero, 1962), como en Belice (Franz, 1991). En Cuba, los murciélagos representaron el $61 \%$ de la dieta (Kirkconnell et al., 1999). En San Paulo, los murciélagos estuvieron presentes en la dieta en proporciones menores (6\%, Motta \& Taddei, 1992; 4\%, Motta-Junior, 2006). En Cayo, Belice, los murciélagos representaron el 19\% (Philips, 2011). No obstante, en nuestro trabajo el bajo número de murciélagos podría estar influenciado por el tamaño muestreal o reemplazado por el alto consumo de aves. Posterior a nuestro período de muestreo, se obtuvieron varias egagrópilas $(c a .10)$ en la misma percha del Parque Arqueológico Rumipamba. En estas se observó que las aves seguían siendo predominantes, pero se encontraron dos cráneos de Rattus rattus (E. Ocaña in litt., 2018), una presa imprevista para Asio stygius, ya que en otros trabajos los roedores están ausentes en su dieta (Lehmann, 1957; Borrero, 1967; Franz, 1991; Motta \& Taddei, 1992; Kirkconnell et al., 1999; Lopes et al., 2004; Motta-Junior, 2006; Phillips, 2011).

Los insectos estuvieron representados solo por escarabajos, la presencia de este ítem en la dieta de Asio stygius cuenta con reportes previos igualmente en proporciones bajas (Motta et al., 1992; Phillips, 2009). Dos autores de esta publicación (Glenda Pozo y Héctor Cadena-Ortíz) han preparado varios especímenes de aves para colecciones científicas y, en su experiencia, los restos de insectos encontrados en el contenido estomacal de paserinos están mucho más fragmentados que los restos encontrados en las egagrópilas de nuestro estudio. Por tal razón, los insectos no fueron considerados como contenido secundario (material sobrante en el estómago de las aves consumidas) en este trabajo. Al contrario, encontramos numerosas semillas en las egagrópilas provenientes de Nayón, las que interpretamos como probable contenido secundario.

El 16 abril 2016, la primera vez que se observó a los jóvenes de Asio stygius en Nayón, presumimos que debieron tener más de un mes de edad, en base a que Lopes et al. (2004) observaron pichones de 27 días aún en el suelo incapaces de perchar en lo alto; siendo el suelo el principal sitio para anidación de $A$. stygius (Motta-Junior et al., 2010). Aunque Kirkconnell et al. (1999) señalan a A. stygius como una rapaz altamente territorial y Lopes et al. (2004) mencionan que luego de la depredación de unos pichones de A. stygius, los adultos fueron observados reiteradamente en la misma área de su nido; después de 22 mayo 2016 no obtuvimos más registros de los búhos, a pesar de reiteradas visitas; cabe mencionar que en el área los dueños realizaron una limpieza de maleza esos días. Kirkconnell et al. (1999) mencionan que podría haber una variación en el color del iris relacionado con la edad en $A$. stygius, cambiando de amarillos en los jóvenes a naranja en los adultos. En la localidad de Nayón, no observamos cambios en la coloración del iris de los jóvenes, mientras que su plumaje fue similar a lo previamente reportado (Bond, 1942; Kirkconnell et al., 1999). 
Asio stygius es una especie excepcionalmente difícil de detectar y con una flexibilidad en el rango de elevación y el hábitat (Miller, 1952; Schmitt et al., 2013). Aunque se conoce muy poco de sus preferencias de hábitat, parece estar asociada a bosques húmedos y semihúmedos, semiáridos, bosques secundarios semiabiertos, áreas agrícolas, parques, plantaciones forestales y ser tolerante a hábitats alterados (Miller, 1952; Ridgely y Greenfield, 2001; König y Weick, 2008; Freile et al., 2012; Schmitt et al., 2013). Son pocos y dispersos los registros de A. stygius a lo largo de su distribución (Enríquez, 2015). En Tamaulipas, México, esta especie se volvió a registrar después de un siglo (Rodríguez-Ruíz y Herrera-Herrera, 2009). Registros de este búho en Perú sugieren que su distribución sería menos fraccionada de lo que se pensaba (Schmitt et al., 2013). En Ecuador, la mayoría de registros y los más recientes son de la provincia de Pichincha, dato que podría estar influenciado por el mayor número de observadores de aves en esta provincia. Precisamente en la ciudad de Quito, Pichincha, existen varios parques donde se ha registrado a A. stygius: Rumipamba, La Carolina, Bicentenario y Guangüiltagua, los que están separados entre sí por 1 a $3 \mathrm{~km}$. Sería óptimo realizar anillamiento o telemetría para constatar si se trata de uno o varios individuos. En trabajos con telemetría para esta especie en Belice, se determinó que una hembra de A. stygius se desplazó cerca de $15 \mathrm{~km}$ forrajeando en un hábitat no urbano (Phillips, 2011).

Asio stygius se distribuye localmente en América desde $600 \mathrm{~m}$ hasta los $3100 \mathrm{~m}$ (König y Weick, 2008). En Ecuador, Ridgely \& Greenfield (2001) mencionan un rango altitudinal para este búho de 1700 a $3100 \mathrm{~m}$, mientras que McMullan \& Navarrete (2017) mencionan 1500 a 3100 m. Nosotros encontramos registros en un rango altitudinal de 27 a $2940 \mathrm{~m}$. De todos los registros que reportamos, 26 fueron georeferenciados confiablemente, y nueve están bajo los $1500 \mathrm{~m}$. Los registros menos esperados son los de las provincias de Guayas y Orellana (Tabla 4). Aún quedan grandes vacíos en su distribución, pues se esperaría que A. stygius esté presente a lo largo de la región andina, pero sus registros son discontinuos. Por ejemplo, no hay registros para las provincias de Carchi, Cotopaxi, Bolívar, Tungurahua y Cañar; mientras que para Chimborazo solo hay un registro histórico (Tabla 4, Fig. 2). Asio stygius continúa siendo una especie con prioridad de investigación. Con mayor número de registros se podría realizar un análisis confiable de uso de hábitat y distribución potencial. En particular, se requiere mayor esfuerzo de muestreo en la serranía centro y se requiere mayor colección de egagrópilas en diferentes áreas y durante un mayor rango temporal para evidenciar patrones.

\section{AGRADECIMIENTOS}

A todos quienes compartieron desinteresadamente sus registros y valiosa información, a los miembros de Aves Quito, Ecuador Birders y Aves_Ecuador, a Walter Guillén Pozo y Orlando Carrión por compartir sus apreciables registros de Guayas, a Cesar Moreno por reportar el búho en el Bicentenario y Edison Ocaña por estar pendiente del búho en Rumipamba, a Sofía Muñoz por la ayuda en la identificación de coleópteros, a Daysi Pachacama la colaboración en las salidas de observación, a Eli Montenegro por la edición de las figuras, a Gorki Ríos-Alvear por diseñar el mapa, a los curadores de los diferentes museos y a los revisores que aportaron en este manuscrito. 


\section{-a.m}

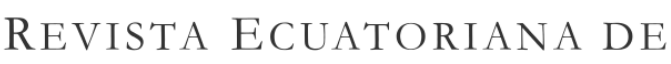

\section{ORNITOLOGÍA}

\section{ARTICULOIARTICLE}

TABLA 4. Registros del Búho Estigio Asio stygius en Ecuador. Las iniciales indican el número de espécimen de los siguientes museos: (AMNH) American Museum of Natural History; (ANSP) Academy of Natural Sciences of Philadelphia; (FMNH) Field Museum of Natural History; (INM-MCN) Instituto Nacional Mejía; Museo De Ciencias Naturales; (MECN) Museo Ecuatoriano de Ciencias Naturales; (MLZ) Moore Laboratory of Zoology; (NRM) Swedish Museum of Natural History; (QCAZ) Museo de Zoología de la Pontificia Universidad Católica del Ecuador; (SBMNH) Santa Barbara Museum of Natural History.

\begin{tabular}{|c|c|c|c|c|c|c|c|c|c|}
\hline Provincia & Localidad & Piso Zoogeográfico & Latitud & Longitud & Altitud & Año & Documentación & Referencia & Notas \\
\hline Azuay & Reserva Yunguilla & Temperado & -3.300 & -79.300 & 1200 & 2010 & auditivo y visual & $\begin{array}{l}\text { Lelis Navarrete in } \\
\text { litt. }\end{array}$ & \multirow{6}{*}{$\begin{array}{l}\text { Un individuo adulto } \\
\text { rescatado junto al } \\
\text { monumento El Choclo, } \\
\text { camino al centro de rescate } \\
\text { el ave muere, por su mala } \\
\text { condición "su ala estaba } \\
\text { desprendida". } \\
\text { Observado por } 1 \text { semana } \\
\text { desde el } 25 \text { de noviembre, } \\
\text { perchado a } 20 \mathrm{~m} \text { en un árbol } \\
\text { "Poma Rosa" en una finca } \\
\text { de Cacao de } 0,02 \mathrm{~km}^{2} \text {, } \\
\text { observado con Jairo Lara. } \\
\text { Fotografiado el } 29 \text { de Julio, } \\
\text { el hábitat es Bosque Seco } \\
\text { Tropical. }\end{array}$} \\
\hline Chimborazo & Cerro Chimborazo & & & & & 1937 & espécimen (FMNH 101746) & VertNet & \\
\hline El Oro & El Faique, Zaruma & Temperado & -3.697 & -79.622 & 940 & 2016 & foto & $\begin{array}{l}\text { Margarita León in } \\
\text { litt. }\end{array}$ & \\
\hline Guayas & $\begin{array}{l}\text { Finca de cacao al } \\
\text { noreste de Reserva } \\
\text { Ecológica Manglares } \\
\text { Churute }\end{array}$ & Tropical Occidental & -2.426 & -79.548 & 30 & 2012 & foto & $\begin{array}{l}\text { Orlando Carrión in } \\
\text { litt. }\end{array}$ & \\
\hline Guayas & $\begin{array}{l}\text { Bosque Protector Cerro } \\
\text { Paraíso, Guayaquil }\end{array}$ & Tropical Occidental & -2.143 & -79.914 & 27 & 2015 & foto & $\begin{array}{l}\text { Walter Guillén in } \\
\text { litt. }\end{array}$ & \\
\hline Imbabura & Hacienda Paramba & Temperado & 0.816 & -78.350 & 690 & 1935 & espécimen (FMNH 100724) & VertNet & \\
\hline Imbabura & $\begin{array}{l}8 \mathrm{~km} \text { al norte de } \\
\text { Salinas }\end{array}$ & Temperado & 0.565 & -78.131 & 1400 & 2012 & Auditivo & Juan Freile in litt. & $\begin{array}{l}\text { Escuchado en compañía de } \\
\text { Niels Krabbe }\end{array}$ \\
\hline Imbabura & Mojanda & & & & & s.f. & NRM & Juan Freile in litt. & \\
\hline Imbabura & Piganta & & & & & s.f. & NRM & Juan Freile in litt. & \\
\hline Loja & Sozoranga & Temperado & -4.350 & -79.783 & 1400 & 1993 & Auditivo & Schmitt et al., 2013 & \\
\hline Loja & Angashcola & & & & & 1994 & Auditivo & Schmitt et al., 2013 & $\begin{array}{l}\text { Las coordenadas reportadas } \\
\text { se ubican en Perú }\end{array}$ \\
\hline Loja & Reserva Utuana & Temperado & -4.367 & -79.717 & 2517 & 2006 & foto & eBird & \\
\hline Loja & Alamor & Temperado & -4.017 & -80.020 & 1328 & 2017 & foto & $\begin{array}{l}\text { Guadalupe Calle in } \\
\text { litt. }\end{array}$ & $\begin{array}{l}\text { Un individuo adulto fue } \\
\text { rescatado de niños que lo } \\
\text { apedreaban en un parque, } \\
\text { fue remitido al zoológico } \\
\text { municipal del Loja, } \\
\text { presentaba fuertes fracturas, } \\
\text { falleció dos días después. }\end{array}$ \\
\hline Orellana & Francisco de Orellana & Subtropical oriental & -0.450 & -77.019 & 281 & 2013 & foto & $\begin{array}{l}\text { Edison Mejia in litt., } \\
\text { https://m.facebook.c }\end{array}$ & $\begin{array}{l}\text { Un espécimen adulto fue } \\
\text { rescatado de una piscina de }\end{array}$ \\
\hline
\end{tabular}

Revista Ecuatoriana de Ornitología, 3, 20-31 


\begin{tabular}{|c|c|c|c|c|c|c|c|c|c|}
\hline & & & & & & & & $\begin{array}{l}\frac{\text { om/MAEPNY/photo }}{\text { S/a.3289301371600 }} \\
\underline{65.88474 .32886972} \\
\underline{3832773 / 513855438} \\
\underline{667533 / ? \text { type }=3 \& \text { th }} \\
\underline{\text { eater }}\end{array}$ & $\begin{array}{l}\text { lixiviados y fue remito al } \\
\text { zoológico municipal de } \\
\text { Francisco de Orellana por } \\
\text { José Narváez. } \\
\text { Aparentemente fue liberado } \\
\text { en las inmediaciones del } \\
\text { zoológico tras recibir } \\
\text { atención veterinaria. }\end{array}$ \\
\hline Pichincha & Hacienda Anagumba & Temperado & 0.200 & -78.399 & 2890 & 1951 & espécimen (ANSP 162889) & Schmitt et al., 2013 & $\begin{array}{l}\text { Etiquetado como provincia } \\
\text { de Imbabura }\end{array}$ \\
\hline Pichincha & Cotocollao & Temperado & -0.113 & -78.501 & 2800 & 2010 & foto & Juan Ríos in litt. & $\begin{array}{l}\text { Fotografiado por Juan Carlos } \\
\text { Ríos \& Sandy Espinosa. }\end{array}$ \\
\hline Pichincha & Rio Blanco, Mindo & $\begin{array}{l}\text { Subtropical } \\
\text { Occidental }\end{array}$ & & & & 1931 & espécimen (MLZ 4704) & VertNet & \\
\hline Pichincha & Cerro del Cayambe & Altoandino & & & & 1940 & $\begin{array}{l}\text { espécimen (INM-MCN } \\
0288 \text { ) }\end{array}$ & $\begin{array}{l}\text { Colección visitada } \\
\text { por los autores. }\end{array}$ & \\
\hline Pichincha & Valle de Zambiza & Temperado & & & & 1940 & espécimen (FMNH 102513) & VertNet & \\
\hline Pichincha & $\begin{array}{l}\text { Montes de Onagunba; } \\
\text { Páramo de Mojanda }\end{array}$ & Altoandino & 0.150 & -78.349 & 2300 & 1946 & $\begin{array}{l}\text { especímenes (SBMNH } 7825 \\
-7827 \text { ) }\end{array}$ & VertNet & \\
\hline Pichincha & Paramo del Iliniza & Altoandino & & & & 1946 & espécimen (SBMNH 7824) & VertNet & \\
\hline Pichincha & Rinconada & & & & & 1946 & espécimen (SBMNH 7823) & VertNet & \\
\hline Pichincha & El Dorado, Quito & Temperado & -0.218 & -78.497 & 2890 & 1982 & espécimen (QCAZ 722) & $\begin{array}{l}\text { Colección visitada } \\
\text { por los autores. }\end{array}$ & \\
\hline Pichincha & Quito & Temperado & & & & 1991 & espécimen (ANSP 183867) & Schmitt et al., 2013 & \\
\hline Pichincha & $\begin{array}{l}\text { Parque Itchimbía, } \\
\text { Quito }\end{array}$ & Temperado & -0.222 & -78.499 & 2890 & 2015 & Foto & Rosero, 2015 & $\begin{array}{l}\text { El espécimen fue entregado } \\
\text { al centro veterinario de la } \\
\text { USFQ, de quienes no } \\
\text { obtuvimos repuesta de su } \\
\text { destino final. }\end{array}$ \\
\hline Pichincha & $\begin{array}{l}\text { Parque Arqueológico } \\
\text { Rumipamba }\end{array}$ & Temperado & -0.180 & -78.499 & 2890 & 2015 & Foto & eBird & $\begin{array}{l}\text { En el mismo parque los } \\
\text { autores recolectamos las } \\
\text { egagrópilas en } 2016\end{array}$ \\
\hline Pichincha & $\begin{array}{l}\text { Parque Bicentenario, } \\
\text { Quito }\end{array}$ & Temperado & -0.145 & -78.485 & 2800 & 2005 & espécimen (MECN 8677) & $\begin{array}{l}\text { Colección visitada } \\
\text { por los autores. }\end{array}$ & $\begin{array}{l}\text { En el mismo parque los } \\
\text { autores recolectamos } \\
\text { información y César Moreno } \\
\text { fotografió un evento de } \\
\text { depredación (Fig. 2) }\end{array}$ \\
\hline Pichincha & $\begin{array}{l}\text { Bosque Protector } \\
\text { Jerusalem }\end{array}$ & Temperado & 0.001 & -78.355 & 2300 & 2007 & Foto & eBird & $\begin{array}{l}\text { En eBird hay más registros } \\
\text { en este parque en los años } \\
2009,2014 \text { y } 2015 \text {. }\end{array}$ \\
\hline Pichincha & $\begin{array}{l}\text { Reserva Geobotánica } \\
\text { Pululahua }\end{array}$ & Temperado & 0.001 & -78.478 & 2880 & 2012 & visual y auditivo & eBird & $\begin{array}{l}\text { En la misma reserva esta } \\
\text { especie ha sido registrada } \\
\text { por Dusan Brinkhuizen en } \\
2013 \text { y por Lelis Navarrete } \\
\text { en } 2015 \text {. }\end{array}$ \\
\hline Pichincha & $\begin{array}{l}\text { Jardín Botánico de } \\
\text { Quito }\end{array}$ & Temperado & 0.186 & -78.485 & 2780 & 2013 & espécimen (MECN 8771) & $\begin{array}{l}\text { Colección visitada } \\
\text { por los autores. }\end{array}$ & $\begin{array}{l}\text { En esta localidad hay } \\
\text { registros auditivos en eBird } \\
\text { para el } 2005 \text { y } 2010 .\end{array}$ \\
\hline Pichincha & Nayón & Temperado & -0.157 & -78.415 & 2350 & 2014 & visual & Luis Irene y eBird & $\begin{array}{l}\text { En la misma localidad los } \\
\text { autores recolectamos }\end{array}$ \\
\hline
\end{tabular}




\begin{tabular}{|c|c|c|c|c|c|c|c|c|c|}
\hline & & & & & & & & & $\begin{array}{l}\text { información para el presente } \\
\text { manuscrito en el } 2016 .\end{array}$ \\
\hline Pichincha & Parque Guápulo, Quito & Temperado & -0.196 & -78.472 & 2630 & 2015 & Visual y foto & $\begin{array}{l}\text { Cisneros-Heredia } e t \\
\text { al., } 2015\end{array}$ & $\begin{array}{l}\text { Hay fotografías de este búho } \\
\text { en el mismo parque en eBird } \\
\text { en el } 2016 \text {. }\end{array}$ \\
\hline Pichincha & $\begin{array}{l}\text { Parque Guanguiltagua, } \\
\text { Quito }\end{array}$ & Temperado & -0.178 & -78.464 & 2940 & $\begin{array}{l}2016 y \\
2010 \\
\end{array}$ & auditivo y visual & $\begin{array}{l}\text { Cristian } \\
\text { Llumiquinga in litt. }\end{array}$ & \\
\hline Pichincha & $\begin{array}{l}\text { Hacienda Santa Rosa, } \\
\text { Pomasqui }\end{array}$ & Temperado & -0.054 & -78.458 & 2480 & 1970 & espécimen (QCAZ 163) & $\begin{array}{l}\text { Colección visitada } \\
\text { por los autores. }\end{array}$ & \\
\hline Pichincha & Santa Rosa, Tumbaco & Temperado & -0.197 & -78.404 & 2300 & $2005-2015$ & visual y auditivo & Juan Freile in litt. & \\
\hline Pichincha & Atahualpa & Temperado & 0.134 & -78.374 & 2260 & s.f. & NRM & Juan Freile in litt. & \\
\hline Pichincha & Verdecocha & Altoandino & & & & s.f. & base de datos WBDB-IBAs & Juan Freile in litt. & \\
\hline Pichincha & Volcán Pichincha & Altoandino & & & & 1962 & espécimen (AMNH 708687) & Schmitt et al., 2013 & \\
\hline
\end{tabular}


OPEN@ACCESS

\section{REFERENCIAS}

eBird. (2016, Noviembre 01) eBird. Version 2016-11-01. BirdAudubon and Cornell Lab of Ornithology. URL: https://ebird.org/ebird/map/

Bond, J. (1942). Notes on the devil owl. The Auk, 59(2), 308-309. DOI:

http://www.dx.doi.org/10.2307/4079570

Borrero, J.I. (1967) Notas sobre hábitos alimentarios de Asio stygius robustus. Hornero, 10(4), 445-447. URL: http://digital.bl.fcen.uba.ar/Download/008_ElHornero/008_ElHornero_v010_n04_articulo445.pdf

Brito, J., Orellana-Vásquez, H., Cadena-Ortiz, H., Vargas, R., Pozo-Zamora, G., \& Curay, J. (2015).

Mamíferos pequeños en la dieta de la lechuza Tyto alba (Strigiformes: Tytonidae) en dos localidades del occidente de Ecuador, con ampliación distribucional de Ichthyomys hydrobates (Rodentia: Cricetidae). Papéis Avulsos de Zoologia, 55(19), 261-268. DOI: http://dx.doi.org/10.1590/0031-1049.2015.55.19

Cadena-Ortiz, H., Freile, J.F., \& Bahamonde-Vinueza, D. (2013). Información sobre la dieta de algunos búhos (Strigidae) del Ecuador. Ornitología Neotropical, 24, 469-474. URL: https://sora.unm.edu/node/133393

Cadena-Ortiz, H., Garzón, C., Villamarín-Cortéz, S., Pozo-Zamora, G.M., Echeverría-Vaca, G., Yánez, J., \& Brito-M, J. (2016). Diet of the Burrowing Owl Athene cunicularia, in two locations of the inter-Andean valley Ecuador. Revista Brasileira de Ornitologia, 24(2), 122-128. URL: http://www4.museugoeldi.br/revistabrornito/revista/index.php/BJO/article/view/1153/pdf_960

Cisneros-Heredia, D.F., Amigo, X., Arias, D., Arteaga, J., Bedoya, J., Espinosa, S., Montenegro, E., Nazati, G., \& Carrión, J.M. (2015). Reporte del 1er Conteo Navideño de Aves de Quito, Ecuador. Avances en Ciencias e Ingenierías, 7(2), B37-B51. DOI: http://dx.doi.org/10.18272/aci.v7i2.256

Enríquez P.L. (2015). Los Búhos Neotropicales: diversidad y conservación. México: Ecosur. Franz, M. (1991). Field observations on the Stygian Owl Asio stygius in Belize, Central America. En Abstracts of presentations made at the annual meeting of The Raptor Research Foundation, Inc., Held at Tulsa, Oklahoma, on 6-10 November 1991. Journal of Raptor Research, 25(4), 151-164. URL: https://sora.unm.edu/sites/default/files/journals/jrr/v025n04/p00151-p00164.pdf

Freile, J.F., Castro, D.F., \& Varela, S. (2012). Estado del conocimiento, distribución y conservación de aves rapaces nocturnas en Ecuador. Ornitología Neotropical, 23, 235-244. URL:

https://sora.unm.edu/sites/default/files/Freile.pdf

Freile, J.F., Guevara, E., Pacheco, C., \& Santander, T. (2015). Los Búhos del Ecuador. En P. L. Enríquez (Ed.), Los Búhos Neotropicales: diversidad y conservación (pp. 333-353). México: Ecosur.

Jaksic, S.M. (1989) What do carnivorous predators cue in on: size or abundance of mammalian prey? A crucial test in California, Chile, and Spain. Revista Chilena de Historia Natural, 62, 237-249. URL: http://rchn.biologiachile.cl/pdfs/1989/2/Jaksic_1989.pdf

Kirkconnell, A., Wechsler, D., \& Bush, C. (1999). Notes on the Stygian owl (Asio stygius siguapa) in Cuba. El Pitirre, 12, 1-3.

König, C., \& Weick, F. (2008). Owls of the world, second edition. London: Christopher Helm. Lehmann, F.C. (1957). Contribuciones al estudio de la fauna de Colombia XII. Novedades Colombianas, 3 , 101-156. URL: 
http://museo.unicauca.edu.co/sites/default/filesfile/N3\%20diciembre\%20de\%201957/ART.1\%20CONTRIBU CIONES\%20AL\%20ESTUDIO\%20DE\%20LA\%20FAUNA\%20DE\%20COLOMBIA\%20XII\%20por\%20F. C.pdf

Lopes, L.E., R. Goes, S. Souza, \& de Melo R.F. (2004). Observations on a nest of the Stygian Owl (Asio stygius) in the central Brazilian cerrado. Ornitologia Neotropical, 15, 423-427. URL:

https://sora.unm.edu/sites/default/files/journals/on/v015n03/p0423-p0428.pdf

McMullan, M., \& Navarrete, L. (2017). Fieldbook of the Birds of Ecuador including the Galapagos Islands and common mammals. Second Edition. Ratty Ediciones. Quito, Ecuador.

Miller, A.H. (1952). Supplemental data on the tropical avifauna of the arid upper Magdalena Valley of Colombia. Auk, 60: 450-457. URL: https://sora.unm.edu/node/20097

Motta, J.C., \& Taddei, V.A. (1992). Bats as prey of Stygian Owls in southeastern Brazil. Journal of Raptor Research, 26(4), 259-260. URL: https://sora.unm.edu/node/53318

Motta-Junior, J.C. (2006). Relações tróficas entre cinco Strigiformes simpátricas na região central do Estado de São Paulo, Brasil. Revista Brasileira de Ornitologia, 14(4), 359-377. URL: http://www4.museugoeldi.br/revistabrornito/revista/index.php/BJO/article/view/2705/pdf 422

Motta-Junior, J.C., Granzinolli, M.A.M., \& Monteiro, A.R. (2010). Miscellaneous ecological notes on Brazilian birds of prey and owls. Biota Neotropica, 10(4), 255-259. DOI: http://dx.doi.org/10.1590/S1676$\underline{06032010000400042}$

Phillips, R. (2011). Studying the Stygian Owl Asio stygius robustus in Mountain Pine Ridge, Belize. Spizaetus 12, 2-6. URL: http://assets.peregrinefund.org/docs/newsletters/Spizaetus-12-English.pdf

Pozo-Zamora, G.M., Brito, J., García, R., Alarcón, I., \& Cadena-Ortiz, H. (2017). Primeras observaciones de la dieta del Búho Orejicorto Asio flammeus bogotensis (Strigiformes: Strigidae) en Pichincha, Ecuador.

Revista Ecuatoriana de Ornitología, 1, 1-7. DOI: http://dx.doi.org/10.18272/reo.v0i1.463

Rodríguez-Ruíz, E. R., \& Herrera-Herrera, J. R. (2009). A un siglo del registro del búho cara oscura (Asio stygius) en el centro de Tamaulipas y notas sobre su distribución en México y los Estados Unidos de América. Huitzil, 10(2), 56-60. URL: http://www.scielo.org.mx/scielo.php?script=sci_arttext\&pid=S1870$\underline{74592009000200004}$

Ridgely, R.S., \& Greenfield, P.J. (2001) The birds of Ecuador: status, distribution, and taxonomy. Ithaca, New York: Cornell University Press.

Rosero, M. (2015, Septiembre 12). Un búho herido fue encontrado en el Itchimbía. Diario El Comercio. URL: http://www.elcomercio.com/tendencias/buho-herido-encontrado-parque-itchimbia.html

Schmitt, C.J., Valqui, T \& Witt, C.C. (2013). The expanding known range of Stygian Owl (Asio stygius) in the Andes. Boletín UNOP 8 (2), 37-45. URL:

http://boletinunop.weebly.com/uploads/6/2/2/6/62265985/bolet\%C3\%ADn_unop_vol._8_n\%C2\%B02. 2013. pdf

VerNet (2016, Noviembre 01). VertNet. Version 2016-09-29. National Science Foundation. URL:

http://portal.vertnet.org/search 